\title{
Terapias neuroprotectoras y neurorestauradoras en el tratamiento de la enfermedad de Parkinson
}

\author{
Neuroprotective and neurorestorative therapies for the treatment of \\ Parkinson's disease
}

\author{
E. Garbayo' ${ }^{1}$ M.S. Aymerich ${ }^{2}$, E. Ansorena ${ }^{1}$, J.L. Lanciego ${ }^{2}$, M.J. Blanco-Prieto ${ }^{1}$
}

\section{RESUMEN}

La enfermedad de Parkinson es la segunda enfermedad neurodegenerativa más común después del Alzheimer. Actualmente se dispone únicamente de terapias sintomáticas que, aunque son muy eficaces en las primeras etapas de la enfermedad poseen a largo plazo considerables efectos secundarios. La terapia ideal sería aquella que permitiese frenar o detener la progresión de la enfermedad. Este es el caso de las terapias neuroprotectoras y neurorestauradoras. De entre todas ellas, la terapia celular y la terapia con factores tróficos tipo GDNF son las que mayores expectativas han generado en la comunidad científica. Aunque ya se ha planteado el uso de GDNF para el tratamiento de la enfermedad de Parkinson, es necesario buscar nuevas estrategias que permitan administrar dicho factor neurotrófico en las zonas concretas del cerebro donde vaya a ejercer su acción. Aquí se discute el uso de micropartículas como el sistema más apropiado para la administración de dicho factor neurotrófico.

Palabras clave. Enfermedad de Parkinson. Neuroprotección. Neuroregeneración. GDNF. Micropartículas.

\begin{abstract}
Parkinson's disease is the second most common neurodegenerative disorder after Alzheimer's disease. Current therapies are symptomatic and, although these therapies are efficacious during the early stages of the disease, they present important side effects when they are used for a long time. The ideal therapy would be the one that would slow down or stop the progression of the disease. This can be achieved, for instance, with neuroprotective and neurorestorative therapies. Among them, cell therapy and therapy with trophic factors such as glial cell line derived neurotrophic factor (GDNF) are the most challenging and promising ones for the scientific community. Although the use of GDNF as a treatment for Parkinson's disease was proposed several years ago, it is necessary to develop alternative strategies to deliver GDNF appropriately to concrete areas of the brain. Here, the use of microspheres as the most suitable tool for the administration of this neurotrophic factor is discussed.
\end{abstract}

Key words. Parkinson's disease. Neuroproteccion. Neuroregeneration. GDNF. Microspheres.
1. Departamento de Farmacia y Tecnología Farmacéutica, Facultad de Farmacia, Universidad de Navarra.

2. Laboratorio de Neuromorfología y Trazadores, CIMA, Universidad de Navarra.

\section{Correspondencia:}

María José Blanco-Prieto

mjblanco@unav.es

Departamento de Farmacia y Tecnología Farmaceútica

Irunlarrea №1

Univesidad de Navarra

31008 Pamplona 


\section{ASPECTOS GENERALES}

Las enfermedades neurodegenerativas son, junto con las enfermedades vasculares y las oncológicas, la principal causa de mortalidad y morbilidad en la población española ${ }^{1}$. La enfermedad de Parkinson (EP) es la segunda enfermedad neurodegenerativa mas frecuente después del Alzheimer ${ }^{2}$ afectando aproximadamente al 15\% de las personas mayores de 65 años. Además, al tratarse de una patología asociada al envejecimiento las probabilidades de adquirir la enfermedad aumentan con la edad. Por otro lado, dado el envejecimiento generalizado de la población, no sería extraño que el número de casos se doblara durante la próxima generación. Por todo ello, resulta necesario buscar nuevas terapias eficaces y seguras que consigan tratar dicha patología.

La EP cursa con la muerte progresiva de las neuronas dopaminérgicas de la sustancia negra pars compacta (SNpc) $)^{3,4}$. La principal consecuencia de esta pérdida neuronal es una marcada disminución en la disponibilidad cerebral de dopamina en el núcleo caudado y en el putamen, lugar donde proyectan las neuronas dopaminérgicas de la SNpc, originándose una disfunción en la regulación de las principales estructuras cerebrales implicadas en el control del movimiento, los ganglios basales.

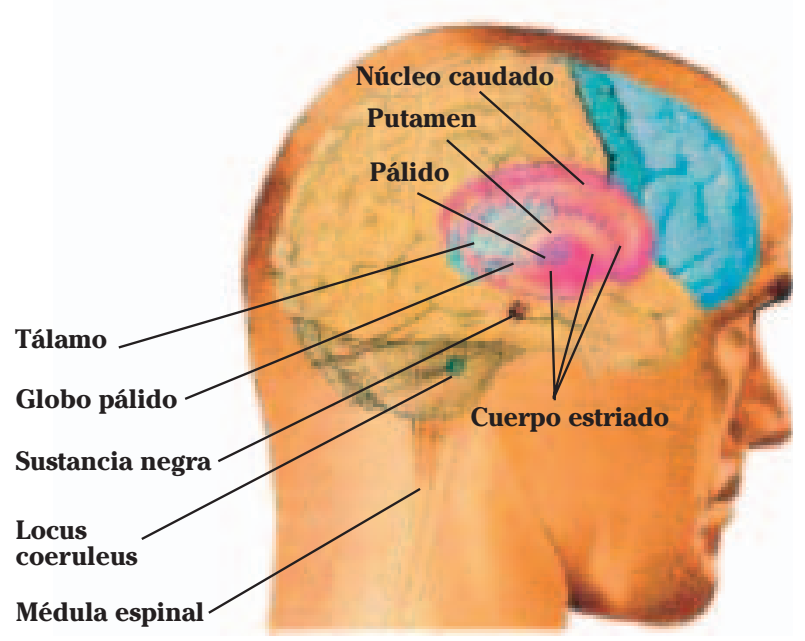

Figura 1. Esquema de la localización de los distintos núcleos de los ganglios basales implicados en el control del movimiento y que se encuentran afectados en la enfermedad de Parkinson.

La EP fue descrita inicialmente en 1817 por James Parkinson, que publicó una ponencia sobre lo que él llamó "la parálisis temblorosa". En ese documento, expone los síntomas principales de la enfermedad que posteriormente llevaría su nombre:

- discinesias: temblor en las manos, brazos, piernas, mandíbula y cara

- rigidez de las extremidades y el tronco 
- bradicinesia o lentitud de movimiento

- inestabilidad postural o problemas de equilibrio

A medida que estos síntomas se hacen más pronunciados, los pacientes tienen dificultad para caminar, hablar y realizar otras tareas simples.

El descubrimiento de la deficiencia de dopamina en el estriado y en la sustancia negra de pacientes con EP supuso un claro avance en el entendimiento de la enfermedad. Posteriormente se demostró la conexión entre la sustancia negra y el estriado, sugiriéndose que la pérdida de neuronas dopaminérgicas en la sustancia negra directamente llevaba a un déficit dopaminérgico en el estriado.

En cuanto al tratamiento de esta enfermedad, el descubrimiento de que la EP cursaba con la pérdida de dopamina permitió desarrollar el primer tratamiento eficaz, la levodopa, a partir del cual se han ido elaborando posteriormente las terapias actuales. Aunque el descubrimiento de la levodopa supuso una revolución en el tratamiento de la EP, pronto se pudo comprobar que, transcurridos 5-10 años de tratamiento, los pacientes desarrollaban fluctuaciones motoras y discinesias. Por lo tanto, posteriormente se trató de entender los mecanismos implicados en la aparición de dichas complicaciones motoras derivadas de la medicación, así como de diseñar nuevas estrategias terapéuticas destinadas a un mejor manejo de los síntomas motores. Actualmente, el trabajo investigador está centrado en la prevención de la degeneración neuronal dopaminérgica. Sin embargo, dado que todavía se desconocen los eventos moleculares que provocan la neurodegeneración todas las terapias actuales se dirigen a tratar los síntomas de la enfermedad y ninguna consigue frenar o detener su progresión.

El agente causante de la EP es desconocido. Se considera que en la mayoría de los casos el origen es multifactorial y la aparición de la enfermedad podría depender de 4 mecanismos: stress oxidativo, predisposición genética, exposición a tóxicos ambientales o del propio organismo y envejecimiento acelerado.

Tampoco existe en la actualidad ningún test que nos permita establecer un diagnóstico definitivo de la enfermedad. Además, el diagnóstico en los estadios tempranos no es sencillo puesto que los primeros síntomas no aparecen hasta que no se produce la pérdida aproximada del $50 \%$ de las neuronas dopaminérgicas de la sustancia negra $^{5}$. La necesidad de diagnosticar la enfermedad en estadios presintomáticos ha llevado a la búsqueda de biomarcadores. Estos biomarcadores deberán ser útiles tanto para mejorar el diagnóstico de la enfermedad como para poder monitorizar su progresión o para demostrar la eficacia clínica de nuevos tratamientos ${ }^{5}$. Actualmente se esta trabajando en el empleo de los siguientes test como biomarcadores $^{6}$ :

- Técnicas de imagen: Neuroimagen funcional (PET y SPECT (Single-photon emisión computed tomography)), ultrasonidos transcraneal, escintigrafía cardíaca...

- Test clínicos: Test afectivos y psicológicos, test neurofisiológicos, test olfatorios, test visuales...

- Test bioquímicos y genéticos: Medida de la concentración sanguínea de sinucleína, malondialdehido, radicales superóxido... 
En este momento, ninguno de los anteriores biomarcadores es capaz de cumplir al mismo tiempo todas las funciones anteriormente citadas por lo que, para poder utilizarlos correctamente resulta necesario conocer para cada caso sus usos y limitaciones ${ }^{5}$.

\section{TRATAMIENTOS ACTUALES}

Los tratamientos actuales comprenden tanto tratamientos farmacológicos como quirúrgicos. A pesar de los avances en el conocimiento de los cambios fisiopatológicos y moleculares desarrollados en la enfermedad, ninguna de estas terapias solas o combinadas consigue modificar el curso clínico de esta devastadora enfermedad $^{7}$.

\section{Tratamientos farmacológicos}

La levodopa es un precursor directo de la dopamina y fue el primer medicamento dopaminérgico empleado en el tratamiento de la EP. Su descubrimiento constituyó uno de los mayores hitos de la neurofarmacología moderna y, tras 3 décadas de uso universal todavía sigue siendo el tratamiento sintomático más eficaz para los pacientes con Parkinson. La administración de dopamina no es posible ya que no atraviesa la barrera hematoencefálica; en cambio, la levodopa (L3,4-Dihidroxifenilalanina) penetra en los ganglios basales, donde es captada por células que poseen transportadores para dicho neurotransmisor y una vez descarboxilada a dopamina, reemplaza el neurotransmisor deficitario. Sin embargo, la administración crónica de este fármaco esta asociada con complicaciones motoras a mediolargo plazo. Para conseguir el efecto necesario cada vez son necesarias dosis más elevadas de fármaco que normalmente también va acompañado de mayores efectos secundarios. Estos efectos secundarios consisten fundamentalmente en fluctuaciones motoras (periodos de mala y buena movilidad alternantes a lo largo del día y en relación a la toma de medicación) y discinesias (movimientos involuntarios) durante la fase de buena movilidad. Los efectos secundarios aparecen a partir de los 2-3 años de iniciarse el tratamiento y, normalmente tras 5 años el $59 \%$ de los pacientes presentan fluctuaciones motoras y el $41 \%$ discinesias.

Para intentar solventar estos problemas, posteriormente se incluyó la combinación de levodopa con un inhibidor de la descarboxilación periférica como carbidopa o benserazide consiguiéndose así que un mayor porcentaje de levodopa penetrase en el cerebro. Similarmente, los inhibidores de la COMT, consiguen prolongar la vida media de la levodopa y de la dopamina, aumentando así el efecto de una dosis dada de levodopa.

Además de aumentar los niveles de los precursores de dopamina, la terapia actual farmacológica está encaminada a limitar la pérdida de dopamina endógena. El inhibidor de MAO-B selegiline actua en este sentido y proporciona considerables beneficios sintomáticos.

Finalmente, el desarrollo de agonistas dopaminergicos que estimulen directamente los receptores dopaminérgicos post-sinápticos reflejan el nacimiento de nuevas terapias surgidas del entendimiento de la enfermedad. 


\section{Tratamientos quirúrgicos}

La cirugía funcional estereotáxica como opción terapéutica en la EP se remonta a 1939. Estas terapias consiguen reducir el temblor y la rigidez. Fueron muy utilizadas antes del descubrimiento de la levodopa pero posteriormente fueron abandonadas cuando el uso de levodopa se hizo más común. El resurgimiento durante los últimos años de este tipo de terapias es debido al reciente entendimiento de la fisiología de los ganglios basales así como al gran desarrollo tecnológico que permite la visualización de distintas estructuras a través de imágenes. Estas imágenes unidas a los registros de la actividad eléctrica de dichas estructuras permiten establecer y caracterizar el área afectada. Por todo ello es posible replantear la cirugía funcional estereotáxica como una opción terapéutica para el tratamiento de la EP.

Actualmente la cirugía funcional estereotáxica está indicada únicamente en aquellos pacientes que no responden bien al tratamiento farmacológico. Los procedimientos que se llevan a cabo son de dos tipos:

- Procedimientos de lesión: palidotomía y talamotomía

- Procedimientos de estimulación (Deep Brain Stimulation; DBS): estimulación talámica, subtalámica y palidal

Sin embargo, aunque estas intervenciones resultan útiles, no son totalmente seguras ni eficaces. En la mayoría de los casos consiguen mejorar únicamente un tipo de síntomas resultando insuficientes como terapias. Además, su uso no consigue en ningún caso frenar la progresión de la enfermedad.

\section{NUEVAS PERSPECTIVAS; TERAPIAS NEUROPROTECTORAS Y NEURORESTAURADORAS}

Ante las grandes limitaciones que ofrecen las terapias actuales, tanto farmacológicas como quirúrgicas, existe la necesidad de desarrollar, con cierta urgencia, otras alternativas que permitan frenar o detener el desarrollo de la enfermedad.

Como ya hemos destacado, aunque las terapias sintomáticas son muy útiles en las primeras etapas de la enfermedad, la calidad de vida de los pacientes va empeorando a medida que la enfermedad progresa y comienza la aparición de los efectos adversos relacionados con la medicación. Por ello, el propósito ultimo de los investigadores debe ser el de bloquear el desarrollo de la enfermedad utilizando fármacos neuroprotectores o neurorestauradores que resulten al mismo tiempo eficaces y seguros. Actualmente han sido identificadas muchas moléculas con potenciales propiedades neuroprotectoras/neurorestauradoras. Este es el caso de la coenzima Q10 o del factor neurotrófico GDNF entre otros. Sin embargo, existen hasta la fecha pocos ensayos clínicos dirigidos a demostrar el efecto neuroprotector/neurorestaurador de estos compuestos.

$\mathrm{Al}$ estudiar este tipo de terapias, muchas veces resulta complicado clasificarlas como neuroprotectoras o como neurorestauradoras ya que normalmente el efecto suele ser una combinación de ambas propiedades. Tal es el caso de la terapia con factores tróficos o de la terapia con células que por si mismas o porque han sido modificadas genéticamente, producen factores neurotróficos. 
A continuación, describiremos más detalladamente la terapia celular y la terapia con factores tróficos, por tratarse de las aproximaciones terapéuticas que mayores expectativas han generado en la comunidad científica para el tratamiento de la EP.

\section{Terapia celular}

El principal objetivo de la terapia celular consiste en reemplazar las células que han degenerado por otras que puedan suplir su función. Normalmente, estas células implantadas en la sustancia negra son células productoras de dopamina y, idealmente, deberían restaurar la conectividad funcional entre la sustancia negra y el estriado.

Además de este papel neurorestaurador, la terapia celular también puede utilizarse con fines neuroprotectores. En este caso deberá utilizarse esta terapia para promover la supervivencia y el rescate de aquellas células que aún no han degenerado.

Hasta la fecha se han utilizado los siguientes tipos celulares:

- Células madre fetales y adultas

- Células de la carótida (productoras de dopamina y de factores neurotróficos) $)^{8,9}$

- Células de médula adrenal (productoras de dopamina)

- Células que liberan factores tróficos y promotores de la supervivencia cuyo fin es evitar que las neuronas que aún no han degenerado mueran. Para ello se han utilizado células de Schwann, astrocitos, células modificadas genéticamente para producir estos factores, etc. ${ }^{10}$

Los inconvenientes más frecuentes que surgen con la terapia celular son infecciones, rechazos en el caso de que las células no sean del propio individuo y degeneración de las propias células implantadas. Existen también otros inconvenientes específicos de cada tipo celular como pueden ser los problemas morales y éticos o el crecimiento incontrolado (teratomas) que surgen con el uso de células madre fetales. Estas células tienen gran capacidad proliferativa pero al mismo tiempo son difíciles de controlar y, hoy por hoy, los resultados en cuanto a su diferenciación son bastante impredecibles. Por otra parte, las células madre adultas no generan tumores, pero tampoco parecen tener el mismo potencial que las células madre embrionarias ya que son inferiores en cuanto a la liberación de neurotransmisores o a sus propiedades eléctricas.

\section{Terapias con factores neurotróficos}

Los factores de crecimiento o factores tróficos son los que, en el desarrollo del embrión, hacen que las primitivas células indiferenciadas se vayan convirtiendo en integrantes del tejido especializado. Los que son necesarios para la supervivencia de las neuronas, se denominan factores neurotróficos ${ }^{11}$.

Los factores neurotróficos consiguen un mantenimiento integral del tejido nervioso, potencian sus recursos celulares y sostienen una conectividad adecuada. También regulan la proliferación, supervivencia, migración y diferenciación de todos los tipos celulares del sistema nervioso además de regular el establecimiento de las cone- 
xiones adecuadas tanto en fases del desarrollo embrionario como en el adulto. Así pues, los factores neurotróficos aparecieron como los candidatos ideales para el desarrollo de terapias neuroprotectoras y neurorestauradoras para el tratamiento de enfermedades neurodegenerativas tipo Alzheimer, esclerosis lateral amiotrófica o enfermedad de Huntington y en concreto para el tratamiento de la EP.

De todos ellos, el GDNF (Fig. 2) es el más apropiado para el tratamiento de dicha patología ya que es el factor neurotrófico dopaminérgico más potente descrito hasta la fecha. Este factor dopaminotrófico por excelencia ha demostrado ser mucho más efectivo que otros factores como el BDNF, TGFb3, NTN, NT4/5, CNTF... ${ }^{12}$.

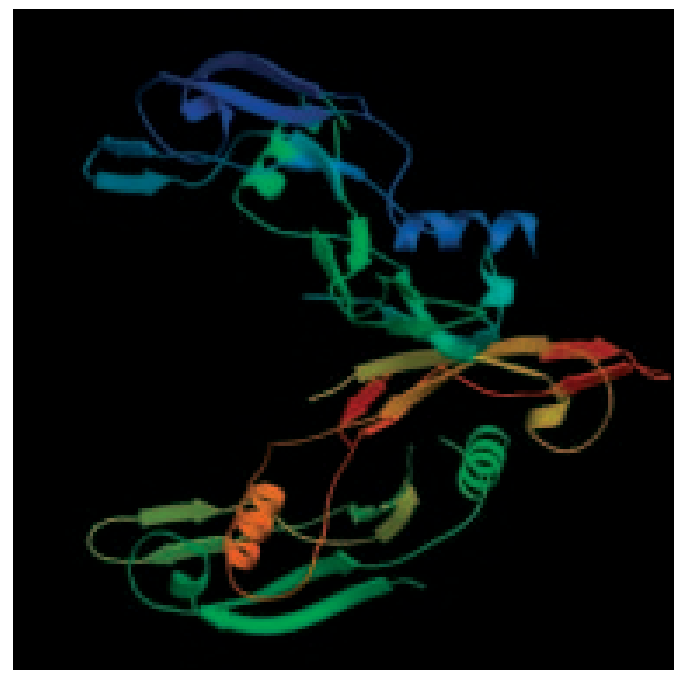

Figura 2. Estructura tridimensional de la molécula de GDNF.

Por ejemplo, el GDNF es capaz de proteger a las neuronas dopaminérgicas de la sustancia negra tanto de la axotomía ${ }^{13}$ como de la degeneración inducida por neurotoxinas tales como la neurotoxina 1metil-4-fenil-1,2,3,6-tetrahidropiridina (MPTP) ${ }^{14}$ o la neurotoxina 6hidroxidopamina (6-OHDA). Por otra parte, la administración exógena de GDNF produce la recuperación funcional en modelos animales (roedores y primates) de enfermedad de Parkinson ${ }^{9,15}$. Además del efecto sobre las neuronas dopaminérgicas mesencefálicas, el GDNF tiene efectos tróficos sobre varios tipos de neuronas del sistema nervioso central y sobre las neuronas sensoriales y autonómicas del sistema nervioso periférico. También promueve la supervivencia de las motoneuronas ${ }^{16} \mathrm{y}$ las protege de la muerte celular o inducida por axotomía ${ }^{17}$.

El uso de GDNF como estrategia terapéutica para el tratamiento de la EP fue propuesto hace años. Al tratarse de una molécula de naturaleza proteica su administración no es sencilla ya que es muy lábil y se degrada con gran facilidad. Además, es incapaz de atravesar la barrera hematoencefálica en cantidad suficiente. Por otra parte, la presencia de receptores distribuidos por el organismo hace que sea preferible su administración de forma local en la zona a tra- 
tar. Para intentar solventar estos problemas han sido elaboradas distintas estrategias encaminadas a liberar GDNF en las zonas estriatales denervadas de dopamina. Estas estrategias abarcan un amplio abanico, que comprende desde diversos implantes celulares ${ }^{18}$ hasta el empleo de vectores virales ${ }^{19}$ de micropartículas ${ }^{20-22}$ y de métodos de infusión directa ${ }^{23}$.

Estudios en roedores y en primates a los cuales se les indujo un cuadro parkinsoniano, señalaron que el GDNF administrado por vía intracerebroventricular aliviaba los síntomas de la enfermedad, promovía el recrecimiento de las neuronas dañadas y aumentaba los niveles de dopamina ${ }^{15}$. Estos resultados dieron lugar a ensayos clínicos en fase I y en fase II para testar en humanos la eficacia y seguridad de la administración de GDNF mediante bombas de infusión ${ }^{24,25}$. Los resultados obtenidos en los distintos ensayos fueron distin$\operatorname{tos}^{26,27}$. Esto puede deberse a diferencias en la dosis de GDNF utilizada, en el catéter y en los métodos de infusión. Además, el sistema elegido para administrar el GDNF no está exento de riesgos ya que puede ocasionar daños en el sitio de implantación y riesgos de infección así como una pobre difusión del factor neurotrófico. Los propios autores del estudio señalaron la necesidad de buscar nuevas estrategias para administrar dicha proteína. De todas ellas, (vectores virales, implantes celulares...) el uso de micropartículas parece ser la estrategia mas apropiada.

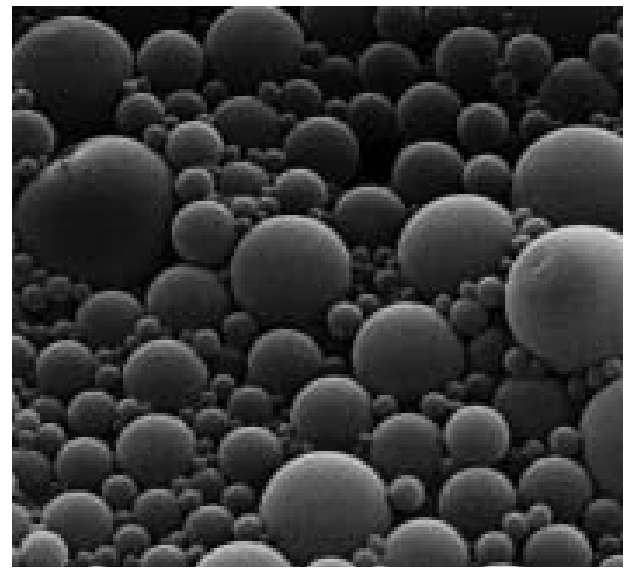

Figura 3. Micropartículas de PLGA (fotografía obtenida por microscopía electrónica de barrido).

Estas nuevas formas farmacéuticas (Fig. 3) permiten aumentar la estabilidad o reducir los efectos adversos del principio activo encapsulado, aunque su principal interés se centra en que son capaces de producir una liberación controlada del medicamento en el lugar designado. Estos sistemas permitirían proteger al GDNF de su degradación así como conseguir la liberación sostenida del mismo durante varios meses. Además, las micropartículas pueden prepararse con polímeros biodegradables tipo PLGA (poly(lactic-co-glycolic acid)) cuyos productos de degradación son totalmente atóxicos y cuya bio- 
compatibilidad con el cerebro ha sido ya totalmente demostrada ${ }^{28}$. Por otra parte, estos sistemas pueden administrarse fácilmente a los pacientes mediante cirugía estereotáxica (Fig. 4), depositando las micropartículas en las zonas concretas del cerebro donde deberán ejercer su acción.

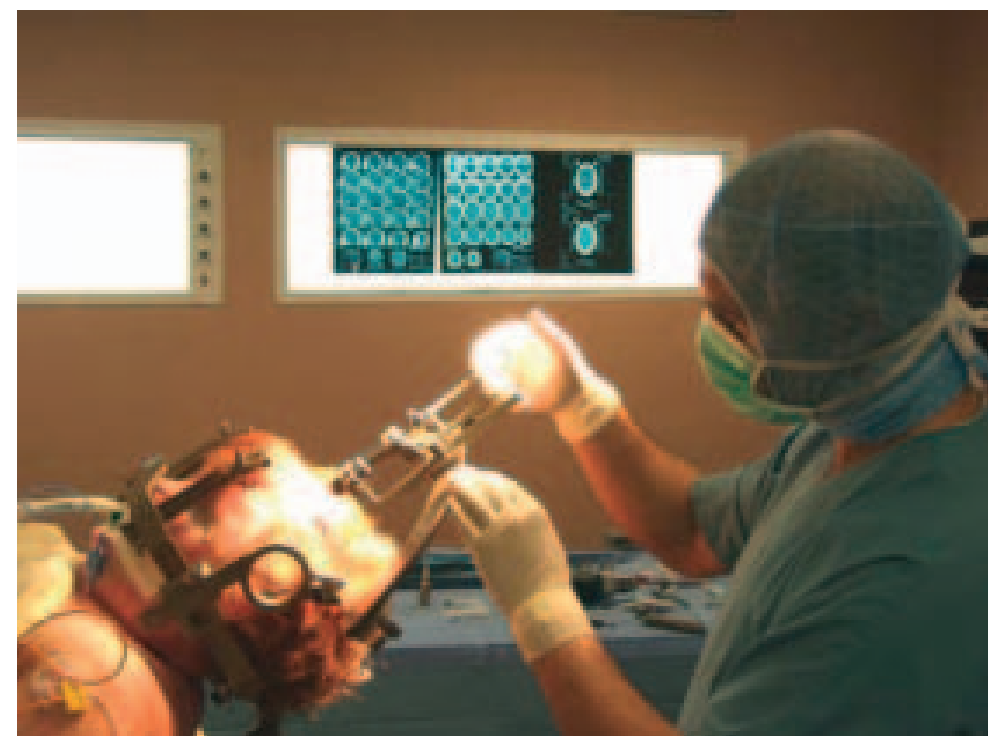

Figura 4. Administración de micropartículas a pacientes mediante cirugía estereotáxica.

La eficacia de estos sistemas particulares cargados con fármacos antitumorales tipo 5-FU y dirigidos al tratamiento de distintos tumores cerebrales está siendo ya estudiada en distintos ensayos clínicos en fase I y II demostrándose que estos sistemas son apropiados para el tratamiento de patologías cerebrales ${ }^{29}$. También se está estudiando la posibilidad de emplear micropartículas de PLGA para administrar fármacos como L-DOPA/Carbidopa ${ }^{30}$, Dopamina ${ }^{31,32}$ o Norepinefri$\mathrm{na}^{31,32}$ con lo que se conseguiría disminuir el número de reacciones adversas y mejorar las terapias actuales. Para revisión ver Garbayo y $\mathrm{col}^{21}$. Además, en cuanto a las terapias con factores tróficos, la eficacia de micropartículas cargados con GDNF se ha estudiado en roedores a los que previamente se les ha ocasionado la enfermedad de Parkinson ${ }^{20,22}$. En nuestro grupo de investigación hemos desarrollado un protocolo de expresión y purificación de GDNF para su microencapsulación en partículas de PLGA. Estás partículas fueron inyectadas mediante cirugía estereotáxica en ratas parkinsonianas. Los resultados obtenidos fueron prometedores ya que pudimos observar una regeneración del tejido dañado en el estriado de esos animales tras la administración y liberación del GDNF a partir de las micropartículas $^{22}$. En un futuro próximo estudiaremos la eficacia de las micropartículas cargadas con GDNF en monos parkinsonianos. Sin duda, los resultados de esta investigación permitirán desvelar si es posible la administración de micropartículas de GDNF para el trata- 
miento de la enfermedad de Parkinson. El empleo con éxito de esta terapia supondría un gran avance en el tratamiento de la enfermedad y marcaría el comienzo de una nueva era en el tratamiento de dicha patología y de las enfermedades neurodegenerativas en general.

\section{AGRADECIMIENTOS}

Al Gobierno de Navarra Departamento de Educación y Departamento de Salud (Proyecto Ortiz de Landázuri).

Fundación MAPFRE Medicina.

\section{BIBLIOGRAFÍA}

1. Segura T, Galindo MF, Rallo-Gutierrez B, Ceña V, Jordán J. Dianas farmacológicas en las enfermedades neurodegenerativas. Rev Neurol 2003; 36: 10471057.

2. Dauer W, Przedborski S. Parkinson's disease: mechanisms and models. Neuron 2003 ; 39: 889-909.

3. Lang AE, Lozano AM. Parkinson's disease. Second of two parts. N Engl J Med 1998; 339: 1130-1143.

4. LANG AE, LOZANo AM. Parkinson's disease. First of two parts. N Engl J Med 1998; 339: 1044-1053.

5. Michell AW, LeWis SJ, Foltynie T, Barker RA. Biomarkers and Parkinson's disease. Brain 2004; 127: 1693-1705.

6. SAVITT JM, DaWSON VL, DAWson TM. Diagnosis and treatment of Parkinson disease: molecules to medicine. J Clin Invest 2006; 116: 1744-1754.

7. Rascol O, Payoux P, Ory F, Ferreira JJ, Brefel-Courbon C, Montastruc Jl. Limitations of current Parkinson's disease therapy. Ann Neurol 2003; 53 Suppl 3: S3-12; discussion S12-15.

8. Espejo EF, Montoro RJ, Armengol JA, Lopez-Barneo J. Cellular and functional recovery of Parkinsonian rats after intrastriatal transplantation of carotid body cell aggregates. Neuron 1998; 20: 197-206.

9. Rosenblad C, Martinez-Serrano A, BJorklund A. Intrastriatal glial cell linederived neurotrophic factor promotes sprouting of spared nigrostriatal dopaminergic afferents and induces recovery of function in a rat model of Parkinson's disease. Neuroscience 1998; 82: 129-137.

10. Shingo T, Date I, Yoshida H, Ohmoto T. Neuroprotective and restorative effects of intrastriatal grafting of encapsulated GDNF-producing cells in a rat model of Parkinson's disease. J Neurosci Res 2002; 69: 946-954.

11. Korsching S. The neurotrophic factor concept: a reexamination. J Neurosci 1993; 13: 2739-2748.

12. Alexi T, Borlongan CV, Faull RL, Williams CE, Clark RG, Gluckman PD et al. Neuroprotective strategies for basal ganglia degeneration: Parkinson's and Huntington's diseases. Prog Neurobiol 2000; 60: 409-470.

13. Beck KD, Valverde J, Alexi T, Poulsen K, Moffat B, Vandlen RA et al. Mesencephalic dopaminergic neurons protected by GDNF from axotomy-induced degeneration in the adult brain. Nature 1995; 373: 339-341.

14. Tomac A, Lindevist E, Lin LF, Ogren SO, Young D, Hoffer BJ et al. Protection and repair of the nigrostriatal dopaminergic system by GDNF in vivo. Nature $1995 ; 373: 335-339$.

15. Gash DM, Zhang Z, Ovadia A, Cass WA, Yi A, Simmerman L et al. Functional recovery in parkinsonian monkeys treated with GDNF. Nature 1996; 380: 252-255. 
16. Henderson CE, Phillips HS, Pollock RA, Davies AM, Lemeulle C, Armanini M et al. GDNF: a potent survival factor for motoneurons present in peripheral nerve and muscle. Science 1994; 266: 1062-1064.

17. Oppenheim RW, Houenou LJ, Johnson JE, Lin LF, Li L, Lo AC et al. Developing motor neurons rescued from programmed and axotomy-induced cell death by GDNF. Nature 1995; 373: 344-346.

18. Kishima H, Poyot T, Bloch J, Dauguet J, Conde F, Dolle F et al. Encapsulated GDNF-producing C2C12 cells for Parkinson's disease: a pre-clinical study in chronic MPTP-treated baboons. Neurobiol Dis 2004; 16: 428-439.

19. MCBRIDE JL, KORDOWER JH. Neuroprotection for Parkinson's disease using viral vector-mediated delivery of GDNF. Prog Brain Res 2002; 138: 421-432.

20. Jollivet C, Aubert-Pouessel A, Clavreul A, Venier-Julienne MC, Remy S, MonTERO-MENEI CN et al. Striatal implantation of GDNF releasing biodegradable microspheres promotes recovery of motor function in a partial model of Parkinson's disease. Biomaterials 2004; 25: 933-942.

21. Garbayo E, Ansorena E, Aymerich MS, Lanciego JL, Blanco-Prieto MJ. Controlled drug delivery to the brain: an overview. Hand Book of Particulate Drug delivery. American Scientific Publishers, NY 2006; En prensa.

22. Garbayo E, Aymerich MS, Ansorena E, Lanciego JL, Blanco-Prieto MJ. Microencapsulated recombinant GDNF: in vitro characterization and in vivo efficacy in a rat model of Parkinson's disease. VIIth Spanish-Portuguese Local Chapter of the Controlled Release Society (SPCL-CRS) Pamplona (Spain) 2006; 85-86.

23. Grondin R, Zhang Z, Yi A, Cass WA, Maswood N, Andersen AH et al. Chronic, controlled GDNF infusion promotes structural and functional recovery in advanced parkinsonian monkeys. Brain 2002; 125: 2191-2201.

24. Gill SS, Patel NK, Hotton GR, O'Sullivan K, McCarter R, Bunnage M et al. Direct brain infusion of glial cell line-derived neurotrophic factor in Parkinson disease. Nat Med 2003; 9: 589-595.

25. Patel NK, Bunnage M, Plaha P, Svendsen CN, Heywood P, Gill SS. Intraputamenal infusion of glial cell line-derived neurotrophic factor in PD: a twoyear outcome study. Ann Neurol 2005; 57: 298-302.

26. Slevin JT, Gerhardt GA, Smith CD, Gash DM, Young AB. Reply: GDNF poses troubling questions for doctors, drug maker. Ann Neurol 2006; 59: 989-990.

27. Sherer TB, Fiske BK, Svendsen CN, LAng AE, Langston JW. Crossroads in GDNF therapy for Parkinson's disease. Mov Disord 2006; 21: 136-141.

28. Veziers J, Lesourd M, Jollivet C, Montero-Menei C, Benoit JP, Menei P. Analysis of brain biocompatibility of drug-releasing biodegradable microspheres by scanning and transmission electron microscopy. J Neurosurg 2001; 95: 489-494.

29. MENEI P, BENOIT JP. Implantable drug-releasing biodegradable microspheres for local treatment of brain glioma. Acta Neurochir Suppl 2003; 88: 51-55.

30. Arica B, Kas HS, Moghdam A, AKalan N, Hincal AA. Carbidopa/levodopa-loaded biodegradable microspheres: in vivo evaluation on experimental Parkinsonism in rats. J Control Release 2005; 102: 689-697.

31. McRae A, DAhlstrom A. Transmitter-loaded polymeric microspheres induce regrowth of dopaminergic nerve terminals in striata of rats with $6-\mathrm{OH}-$ DA induced parkinsonism. Neurochem Int 1994; 25: 27-33.

32. McRae A, Ling EA, Huorth S, Dahlstrom A, Mason D, Tice T. Catecholaminecontaining biodegradable microsphere implants as a novel approach in the treatment of CNS neurodegenerative disease. A review of experimental studies in DA-lesioned rats. Mol Neurobiol 1994; 9: 191-205. 